\title{
Using Topology Optimization to Numerically Improve Barriers to Reverse Engineering
}

Devin Donald LeBaron

Brigham Young University - Provo

Follow this and additional works at: https://scholarsarchive.byu.edu/etd

Part of the Mechanical Engineering Commons

\section{BYU ScholarsArchive Citation}

LeBaron, Devin Donald, "Using Topology Optimization to Numerically Improve Barriers to Reverse Engineering" (2013). Theses and Dissertations. 3787.

https://scholarsarchive.byu.edu/etd/3787

This Thesis is brought to you for free and open access by BYU ScholarsArchive. It has been accepted for inclusion in Theses and Dissertations by an authorized administrator of BYU ScholarsArchive. For more information, please contact scholarsarchive@byu.edu, ellen_amatangelo@byu.edu. 
Using Topology Optimization to Numerically Improve

Barriers to Reverse Engineering

Devin D. LeBaron

A thesis submitted to the faculty of

Brigham Young University

in partial fulfillment of the requirements for the degree of

Master of Science

Christopher A. Mattson, Chair

C. Greg Jensen

Eric R. Homer

Department of Mechanical Engineering

Brigham Young University

May 2013

Copyright $@ 2013$ Devin D. LeBaron

All Rights Reserved 


\author{
ABSTRACT \\ Using Topology Optimization to Numerically Improve \\ Barriers to Reverse Engineering \\ Devin D. LeBaron \\ Department of Mechanical Engineering, BYU \\ Master of Science
}

Here explored is a method by which designers can use the tool of topology optimization to numerically improve barriers to reverse engineering. Recently developed metrics, which characterize the time $(T)$ to reverse engineer a product, enable this optimization. A key parameter used in the calculation of $T$ is information content $(K)$. The method presented in this thesis pursues traditional topology optimization objectives while simultaneously maximizing $K$, and thus $T$, in the resulting topology. This thesis presents new algorithms to 1) evaluate $K$ for any topology, 2) increase $K$ for a topology by manipulating macro-scale geometry and micro-scale crystallographic information for each element, and 3) simultaneously maximize $K$ and minimize structural compliance (a traditional topology optimization objective). These algorithms lead designers to desirable topologies with increased barriers to reverse engineering. It is concluded that barriers to reverse engineering can indeed be increased without sacrificing the desirable structural characteristic of compliance. This has been shown through the example of a novel electrical contact for a consumer electronics product.

Keywords: reverse engineering, topology optimization, barriers to reverse engineering 


\section{ACKNOWLEDGMENTS}

I am thankful for all those who have supported and befriended me as I have worked on this research.

I would like to express thanks to Dr. Mattson for all he has taught me. I have had many opportunities at BYU to work closely with Dr. Mattson and it has been a highlight in my education. He is an excellent engineer, person, and friend and I am honored to have worked so much with him.

I would also like to thank all the members of the Design Exploration Research Group that have helped me in my research and education. I would like to individually thank Kevin Francis who worked closely with me on this particular research. Friendships that I have made in this lab have made my time at BYU as a graduate student very enjoyable.

I would like to acknowledge the help given to me by Dr. Homer, Dr. Balling, and Dr. Parkinson who took the time to meet with me and guide me through some of the hardest parts of my research.

I want to thank my wife Brittany for her love and support.

I am very thankful that I had the opportunity to learn so much here at BYU from so many great individuals. 


\section{TABLE OF CONTENTS}

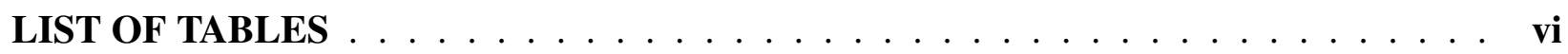

LIST OF FIGURES $\ldots \ldots \ldots \ldots \ldots \ldots \ldots \ldots \ldots$ viii

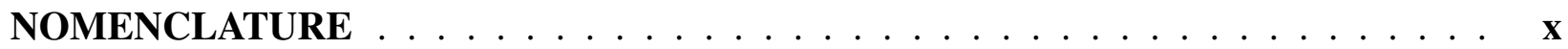

Chapter 1 Introduction . . . . . . . . . . . . . . . . . 1

1.1 Introduction and Background $\ldots \ldots \ldots \ldots \ldots \ldots$

Chapter 2 Literature Review $\ldots \ldots \ldots \ldots \ldots \ldots$

2.1 Topology Optimization . . . . . . . . . . . . . . . . . 5

2.2 Microstructure Sensitive Design $\ldots \ldots \ldots \ldots \ldots$

Chapter 3 New Developments for Increasing Barriers to Reverse Engineering . . . . 9

3.1 Optimization Problem Formulation . . . . . . . . . . . . . . . . . . 9

3.2 Quantification of Information Content $(K)$. . . . . . . . . . . . . . . . . . . 10

3.3 How Information Content is Increased: . . . . . . . . . . . . . . . . . . . . . 13

3.3.1 Geometric Complexity . . . . . . . . . . . . . . . . . . . 13

3.3.2 Microstructure Orientation . . . . . . . . . . . . . . . . . . . 16

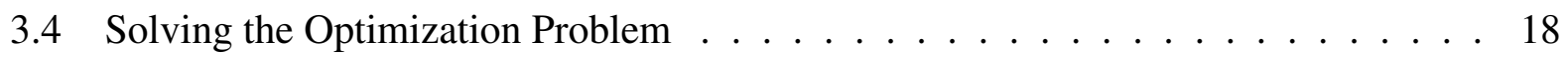

3.4.1 Optimum Void Location and Size . . . . . . . . . . . . . . . . . . . 18

3.4.2 Optimal Microstructure Orientation . . . . . . . . . . . . . . . 20

Chapter 4 Examples . . . . . . . . . . . . . . . . . . . . . . . . 27

4.1 Case Study: Electrical Contact for a Consumer Electronics Product . . . . . . . . . 27

Chapter 5 Conclusions and Future Work . . . . . . . . . . . . . . . . . . 33

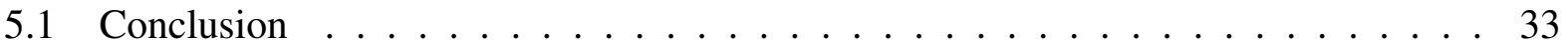

5.2 Future Work . . . . . . . . . . . . . . . . . . . . . 33

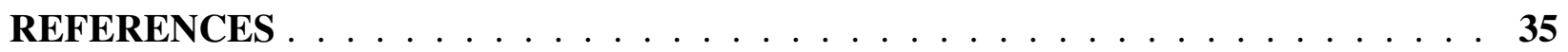




\section{LIST OF TABLES}

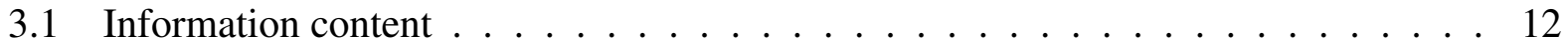

4.1 Comparison of information content $(K)$ and deflection in the different cases $\ldots . .29$ 


\section{LIST OF FIGURES}

3.1 Design domain used for all the examples in Chapter 3. Dimensions are 60 units in the horizontal direction and 30 units in the vertical direction. The loads and boundary conditions in the design domain correspond to half the MBB-beam. . . . 11

3.2 Benchmark A. This is the solution to the topology optimization problem without attempting to add information content. This will be used for comparison to other

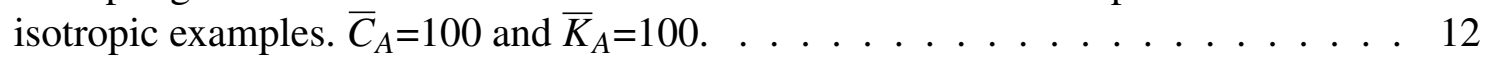

3.3 Convex hull representation of voids in Fig. $3.2 \ldots \ldots$. . . . . . . . . . . 13

3.4 An example of using different microstructure orientations in a topology. Each unique orientation adds 1 piece of information content. In this example information content $(K)$ has been increased by $7 \ldots \ldots \ldots$. . . . . . . . . . . 14

3.5 Design domain with 5 equally spaced voids in an attempt to complicate the geometry in the topology . . . . . . . . . . . . . . . . 15

3.6 Resulting topology using the design domain in Fig. 3.5. $\bar{C}_{A}=125$ and $\bar{K}_{A}=234$. Where $\bar{C}_{A}=\left(\frac{C}{C_{A}} * 100\right)$ and $\bar{K}_{A}=\left(\frac{K}{K_{A}} * 100\right) . C$ and $K$ are evaluated from this topology, while $C_{A}$ and $K_{A}$ represent the $C$ and $K$ of the benchmark topology shown in Fig. 3.2. . . . . . . . . . . . . . . . . . . . .

3.7 Benchmark B. A topology optimization using a uniformly oriented anisotropic material. $\bar{C}_{B}=100$ and $\bar{K}_{B}=100$. Examples using an anisotropic material will be compared to this benchmark. . . . . . . . . . . . . .

3.8 A topology optimization result using an anisotropic material that is optimally oriented at each element. $\bar{C}_{B}=78$ and $\bar{K}_{B}=115 \ldots \ldots \ldots \ldots \ldots$

3.9 Resulting topology with optimum array of voids. $C_{a}=126$ and $K_{b}=277 \ldots \ldots$

3.10 Resulting topology with optimum location of one void to maximize information content and minimize compliance . . . . . . . . . . . . . . 20

3.11 Resulting topology from using four voids and allowing them to move their location and size to find an optimum solution. $\bar{C}_{A}=134$ and $\bar{K}_{A}=192 \ldots \ldots \ldots 21$

3.12 Design domain with one element highlighted. This elements microstructure orientation is determined by the value of $\Theta \ldots \ldots \ldots \ldots . \ldots \ldots 21$

3.135 elements with loads and boundary conditions used for testing the compliance at different microstructure orientations . . . . . . . . . . . . . . 23

3.14 These graphs represent the compliance vs. orientation for the elements shown in

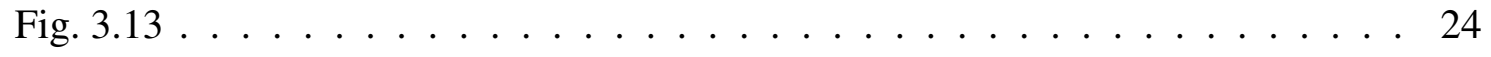

4.1 Design domain for the novel electrical contact . . . . . . . . . . . . 28

4.2 Case 1:(Benchmark C) Uniformly oriented Topology with no forced voids. $\bar{C}_{C}=100$

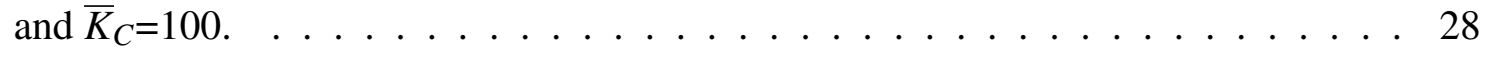

4.3 Case 2: Uniformly oriented topology with 4 forced voids that have been optimally located and sized. $\bar{C}_{C}=109.5$ and $\bar{K}_{C}=192 \ldots \ldots \ldots$. . . . . . . . . . . . . . 29

4.4 Case 3: Optimally oriented topology with no forced voids initially in the design

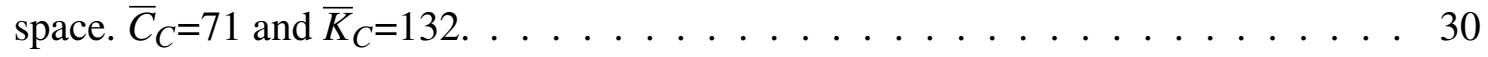

4.5 Case 4: Optimally oriented topology with 4 forced voids that have been optimally

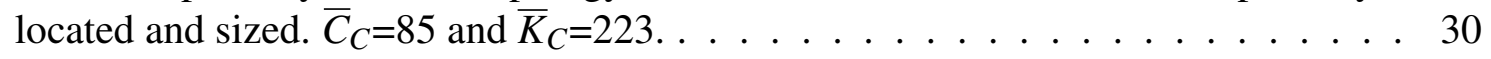


4.6 3D Model of the contact design in Case $4 \ldots \ldots$. . . . . . . . . . . 31

4.7 3D Model of a connector using the contact design in Case $4 \ldots \ldots$. . . . . . . . 31 


\section{NOMENCLATURE}

$T \quad$ Time to Reverse Engineer a Product

$K \quad$ Information Content

$K_{0} \quad$ Initial Information Content

$k \quad$ Stiffness Matrix

$u \quad$ Displacement Vector

$f \quad$ Force Vector

$V_{x} \quad$ Material Volume

$V_{0} \quad$ Design Domain Volume

$v \quad$ Material Volume Fraction $\left(V_{x} / V_{0}\right)$

$x \quad$ Vector of Element Densities

$x_{\min } \quad$ Vector of Minimum Element Densities

$C \quad$ Average Elemental Compliance

$\Theta \quad$ Microstructure Orientation Angle

[ ] Normalized Value of [ ]

$B \quad$ Barrier to extract information about a product from the product itself

$D \quad$ Constitutive Matrix

$B \quad$ Element Shape Function Matrix

$S \quad$ Stress Vector

$T$ Transformation Matrix 


\section{CHAPTER 1. INTRODUCTION}

\subsection{Introduction and Background}

Innovative products are reverse engineered - often by competitors seeking to capture market share. The share gained is directly related to the time it takes to enter the market [1]. Therefore, it is beneficial for innovating companies to impede the reverse engineering efforts of others. This thesis shows that incorporating macro and micro-scale barriers within products will increase the time required to reverse engineer them, presumably delaying competitors' market entry.

Various ways to impede reverse engineering have been explored. Methods include: avoiding explicit disclosure of information [2], creating anti-robust designs [2], designing components that are difficult to access [3], designing components that require unique tools or information [3], and designing components that self destruct when tampered with [3]. In this thesis, the use of topology optimization to impede reverse engineering is explored.

Metrics to define and evaluate barriers to reverse engineering have recently been developed $[4,5]$. The ability to quantify these barriers enables their implementation into topology optimization and other numerical optimization frameworks. Here a brief review of the definitions and metrics created for barriers to reverse engineering will be given, with an emphasis on how they can be used in protecting innovative products.

A barrier to reverse engineering has been defined as anything that impedes the extraction of information about a product from the product itself [4]. The metrics are summarized below in the equations for $B$, the barrier to reverse engineering, and $T$, the time to reverse engineer a product [5].

$$
\begin{gathered}
B=P / F^{2} \\
T=-B S \ln \left(K / K_{0}\right)
\end{gathered}
$$


where $P$ is the power (work per time to extract information), $F$ is the rate of information extraction, $K$ is the information content, and $S$ is the information storage capacity. $B$ and $T$ are related but distinct as described in Harston and Mattson [5]. Often the goal in creating barriers to reverse engineering is to delay the competition until the market is saturated [6]. Thus, the focus is on the maximization of $T$. Increasing $T$ can be accomplished through increasing $B, S$, and/or $K$. Within a topology optimization framework, the information content $(K)$ is related only to the product itself and not to the individual who is reverse engineering it (as is the case for $B$ and $S$ ). Therefore, $K$ is generally evaluated and can be automatically extracted for any generated topology. In this thesis, $T$ is indirectly maximized by maximizing $K$.

Although these metrics now allow a numerical quantification of $K$, in order to make this value a variable in a topology optimization routine it is necessary to automatically extract it. Otherwise the topology optimization would have to pause at each iteration and have the designer calculate and input the new value for the $K$. As topology optimization is already close to a prohibitively long process, this would not be feasible. This has lead to the development of an algorithm that automatically extracts the information content $(K)$ in structures identified during topology optimization iterations. This method is discussed further in Chapter 3.

The information content $(K)$ is the sum of the information content in several categories within a product [5]. By increasing the information content in any individual category the overall information content will be increased. This thesis focuses on increasing the information content in the macro-scale geometry and in the microstructure. Both of these categories are increased using algorithms that work in conjunction with a topology optimization algorithm. Essential to increasing the information content in the microstructure is an understanding of microstructure sensitive design. Both topology optimization and microstructure sensitive design will be discussed more in Chapter 2.

Thesis objectives:

- Develop an algorithm that automatically evaluates the information content $(K)$ in any topology within a topology optimization routine. 
- Within a topology optimization routine, develop algorithms that increase the information content $(K)$ in the macro-scale geometry and micro-scale crystallography of the topology, while maintaining traditional topology optimization objectives.

The remainder of this thesis is outlined as follows: Chapter 2 conducts a literature survey on the enabling technologies used in this thesis, namely topology optimization and microstructure sensitive design. In Chapter 3, algorithms developed to evaluate and increase information content $(K)$ within a topology are presented. In Chapter 4, examples of information content $(K)$ being increased using topology optimization are shown, particularly through the design of a novel electrical contact for a consumer electronics product. In Chapter 5, conclusions are presented. 


\section{CHAPTER 2. LITERATURE REVIEW}

Before presenting our approach for simultaneously optimizing topology and reverse engineering objectives, we must first provide a brief description of two supporting technologies; topology optimization and microstructure sensitive design.

\subsection{Topology Optimization}

Topology optimization is a design tool that distributes material in an optimal lay-out within a design domain. Topology optimization's most common use is for the optimal lay-out of isotropic material in linear-elastic structural problems. In this scenario, the known values are the loads, support conditions, and volume of the structure [7]. Although structural applications of this design tool are the most common, it has been used to optimize performance in a variety of other categories such as, thermal expansion [8], compliant mechanisms [9] [10], piezoelectric surfaces [11], electromagnetic properties [12], material selection [13], and heat transfer [14] [15], to name a few. To the author's best knowledge, topology optimization has not yet been described in the literature as a means to impede reverse engineering. This thesis uses topology optimization to optimize structural characteristics as well as barriers to reverse engineering.

There is a variety of methods that have been developed to perform topology optimization. The most common is the Solid Isotropic Microstructure with Penalization (SIMP) method. This method was first proposed in 1989 by Bendsoe [16]. With a few exceptions all the commercial topology optimization software packages use this method [17]. Other methods that have been developed include the Evolutionary Structural Optimization (ESO) method, the Bi-directional Evolutionary Structural Optimization (BESO) method, derivative based methods and level-set methods. The SIMP method and the level-set method will be discussed further in this thesis.

The SIMP method is also referred to as the "Power-Law Approach". In this method the design domain is discretized with elements having constant material properties. Each element 
also is given a relative density value. These relative density values become the variables in the optimization problem. Each element's material properties become defined as the relative density raised to some power times the material property [18]. This is a finite element based method and has been used in many applications. This approach also requires filtering techniques to ensure that a solution is found. Hundreds of papers have been written on the SIMP method and varieties of the SIMP method. This thesis uses the SIMP method in conjunction with other algorithms to complete its objectives.

More recently, level-set methods of Topology optimization have been developed [19] [20]. These methods allow the designer to select the number of voids initially in the design domain. This would be useful for our application because (as described in Section 3.3) our method artificially introduces voids within the design domain as a means to increase information content. Level-set methods were not used in this thesis because the SIMP method gives more control of the location of the introduced voids. However, implementation of the level-set method in the future could prove more effective at introducing barriers to reverse engineering.

\subsection{Microstructure Sensitive Design}

Microstructure sensitive design is the process of establishing location and orientation of microstructure types within a part or component to attain desired performance [21]. Material microstructure refers to the organization of the crystalline grain structures in a material. For anisotropic materials, the microstructure is such that the material properties vary in different directions. In this thesis, microstructure sensitive design is used to orient individual anisotropic elements in a topology to change its overall compliance. We recognize that there are no common manufacturing practices to manipulate the microstructure of each element individually - although previous work has suggested some ideas to accomplish this [22]. The present thesis focuses on the theories of impeding reverse engineering and not necessarily on manufacturing feasibility.

Within microstructure sensitive design are two important constructs; the microstructure hull and the properties closure. The microstructure hull is the set of all possible microstructures that could exist within a material. The properties closure defines all the different material properties that are attainable with any combination of microstructures within the microstructure hull. Thus, in theory one could obtain any material property within the properties closure using microstructure 
sensitive design [21]. Thus a product could be developed with a performance that could not be recreated without an understanding of the material microstructure.

For clarity, this thesis does not offer contributions to the fields of topology optimization nor microstructure sensitive design, rather it offers contributions to the field of impeding reverse engineering. Namely this thesis introduces algorithms to 1$)$ evaluate information content $(K)$ for any topology, 2) increase $K$ for a topology by manipulating macro-scale geometry and microstructure for each element, and 3) simultaneously maximize $K$ and minimize structural compliance. 


\section{CHAPTER 3. NEW DEVELOPMENTS FOR INCREASING BARRIERS TO REVERSE ENGINEERING}

This Chapter presents new developments that enable topology optimization to be used to numerically increase barriers to reverse engineering. An optimization problem formulation is provided, and a means to quantify the information content and maximize it - for any topology - is presented.

\subsection{Optimization Problem Formulation}

In order to maximize information content while minimizing compliance the following multi-objective problem formulation is used:

$$
\min _{x}\{C(x),-K(x)\}
$$

subject to:

$$
\begin{gathered}
V_{x} / V_{0}=v \\
k u=f \\
0<x_{\text {min }} \leq x \leq 1 \\
0 \leq \Theta \leq 360
\end{gathered}
$$

where $C$ is the structural compliance; $K$ is the information content; $x$ is a vector of element densities; $x_{\min }$ is a vector of minimum densities for the structural elements; $k, u$ and $f$ are the stiffness matrix, displacement vectors, and force vectors, respectively; $V_{x}$ and $V_{0}$ are the material volume and design domain volume; $v$ is the volume fraction; and $\Theta$ is the microstructure orientation of each element. Additional constraints related to $C$ are discussed in Sigmund et al [18]. 
There are two approaches to solve this problem, or any multi-objective optimization problem. The first is integrated generating and choosing [23]. The second is generate first, choose later [24]. Although integrated generating and choosing is used for the remainder of this thesis, either approach can be used to implement the concepts herein.

\section{Topology Optimization Set Up:}

A modified version of the 99-line topology optimization code developed by Sigmund et al [18] is used in this thesis. This code allows the designer to define a two-dimensional design domain with boundary conditions and loads. Its objective is to minimize compliance for a given volume fraction. For full details on this code the reader is referred to Sigmund et al [18]. The design domain of the examples presented in this Chapter is constrained to half of the MBB-beam (simply supported beam) as shown in Fig. 3.1, and the dimensions are 60 units in the horizontal direction, 30 in the vertical direction, and the volume fraction is 0.5 .

When comparing topology optimization results in this thesis, the variables $\bar{C}_{i}$ and $\bar{K}_{i}$ will be used. These variables are the average elemental compliance $(C)$ and information content $(K)$ normalized with respect to a benchmark design. The subscript $i$ refers to which benchmark the topology is being compared to. The equations for $\bar{C}_{i}$ and $\bar{K}_{i}$ are shown below.

$$
\begin{aligned}
\bar{C}_{i} & =\left(\frac{C}{C_{i}} * 100\right) \\
\bar{K}_{i} & =\left(\frac{K}{K}_{i} * 100\right)
\end{aligned}
$$

where $C$ and $K$ are evaluated for the current topology, and $C_{i}$ and $K_{i}$ represent the $C$ and $K$ of the benchmark topology.

\subsection{Quantification of Information Content $(K)$ :}

The information content in a product is the collection of information from different categories such as material, geometry, microstructure, electrical conductivity, color, and other types of information [5]. This thesis focuses only on increasing the information content $(K)$ in the geometry and microstructure categories. To accomplish this, the information content must first be quantified. For geometry, the quantity of information content is the amount of data required to define the ge- 


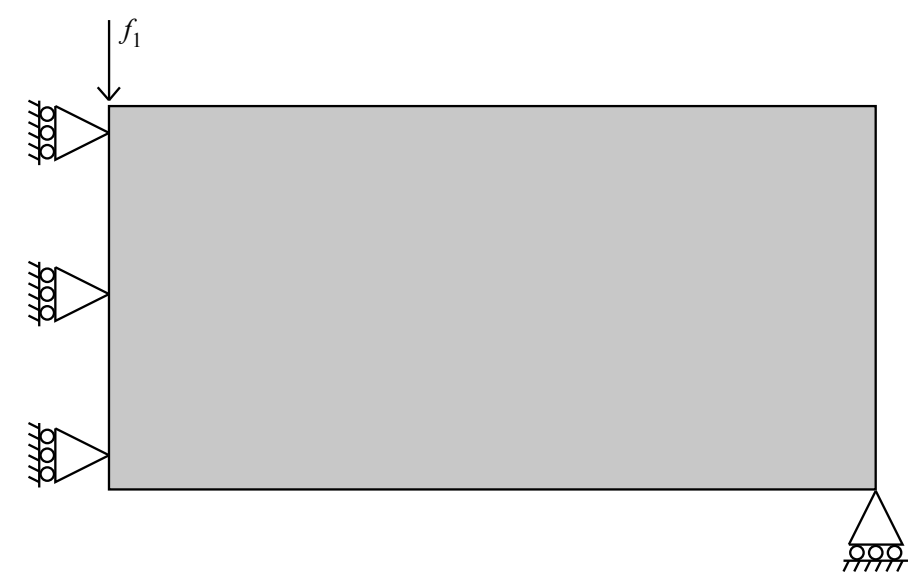

Figure 3.1: Design domain used for all the examples in Chapter 3. Dimensions are 60 units in the horizontal direction and 30 units in the vertical direction. The loads and boundary conditions in the design domain correspond to half the MBB-beam.

ometry. For microstructure, it is the number of distinct microstructures in a given topology. This section describes the algorithm developed to quantify the information content $(K)$ in any topology.

To determine the geometric information content in a topology, the geometry is first decomposed into smaller constructs. To do so, all boundaries between material and the void (empty) regions in the design domain must be geometrically articulated. These boundaries can be represented by polygons as seen in Fig. 3.3. Each vertex of a polygon requires 2 data to be defined in a two dimensional setting. As the geometry is fully described by these polygons, two times the quantity of polygons' vertices is the quantity of data required to reproduce the topology.

Although quantifying the geometric information content in a topology can easily be done manually, the task needs to be automated to avoid interrupting the optimization routine at each topological iteration. In order to automate this process, Matlab's digital image processing function convex hull is used. The convex hull function is able to represent the void regions in the design domain as polygons. These polygons are then used to calculate the amount of geometric information content as described above. Table 3.1 shows the results of the algorithm quantifying the geometric information content for the polygons shown in Fig. 3.2.

A drawback of the convex hull function is its inability to deal with either curvature or protrusions into the convex regions as defined by the hull. Such an inability is also shown in Fig. 3.3, 
polygon D. To help minimize the effect of this drawback an additional step is introduced to this algorithm. The topology is rotated 45 degrees and 90 degrees. At each orientation the geometric information content is quantified using the method described for both the topology and the negative image of the topology. Some orientations allow the convex hull function to better articulate the empty spaces in the topology. Hence, by taking the maximum of the set of information content in these orientations a more accurate quantification of the geometric information content in the topology is obtained.

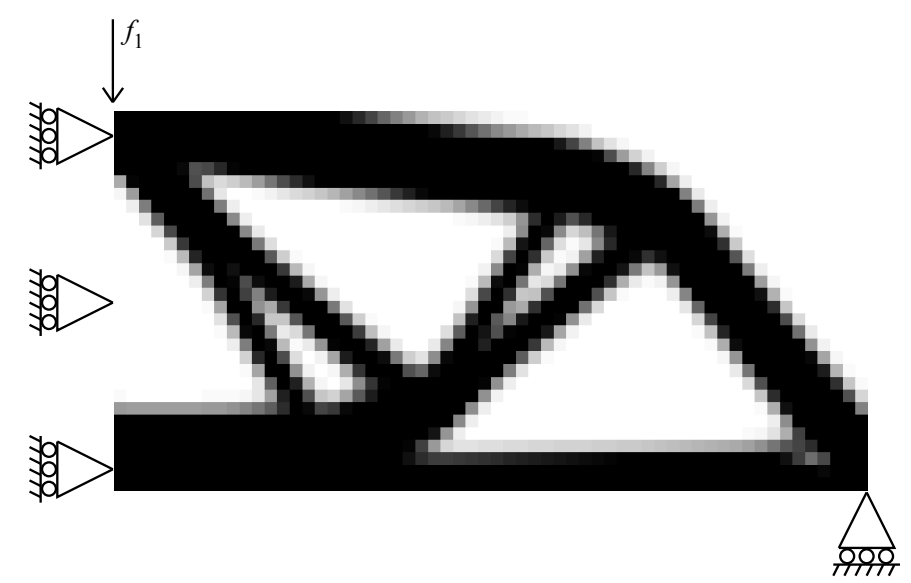

Figure 3.2: Benchmark A. This is the solution to the topology optimization problem without attempting to add information content. This will be used for comparison to other isotropic examples. $\bar{C}_{A}=100$ and $\bar{K}_{A}=100$.

Table 3.1: Information content

\begin{tabular}{lc}
\hline \hline Method & Information \\
\hline Algorithm & 39 \\
\hline A & 6 \\
B & 4 \\
C & 8 \\
D & 4 \\
E & 8 \\
F & 6 \\
TOTAL Hand Calc. & 36 \\
\hline \hline
\end{tabular}




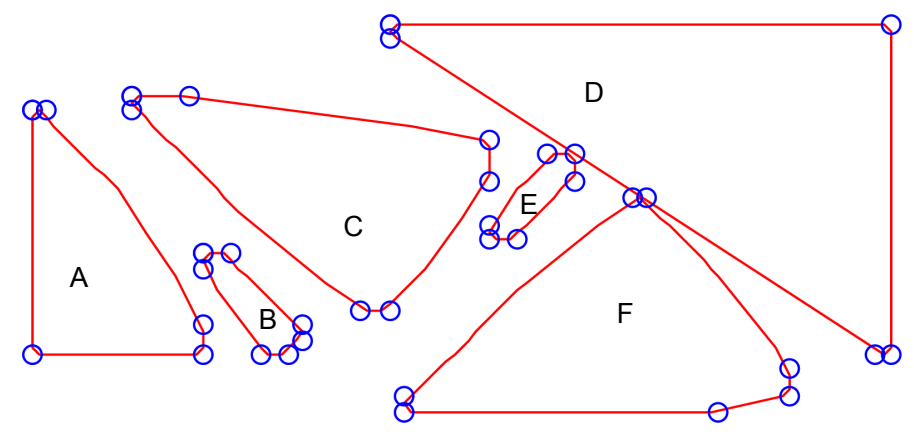

Figure 3.3: Convex hull representation of voids in Fig. 3.2

When quantifying the information content due to the microstructure, it is not necessary to evaluate the orientation of every element in a topology. In general, the orientation of elements in the same sections of the topology tend to be similar (within 5 degrees). Therefore the number of unique microstructures will often be less then the number of elements in the design domain. This grouping of similar orientations can be seen in Fig. 3.4. In this thesis, similar orientations have been defined to be orientations that are within 10 degrees of each other. The information content due to the microstructure is equal to the number of unique microstructure groups in a topology.

\subsection{How Information Content is Increased:}

Increasing the information content in a topology has been accomplished through increasing the geometric complexity and by allowing the microstructure orientation of each element to change. Each is now discussed.

\subsubsection{Geometric Complexity}

The more geometric complexity in a topology, the greater the amount of information content $(K)$. Hence maximizing geometric complexity becomes an objective. Under a topology optimization framework, geometric complexity can only be influenced by addition or subtraction of material within the design domain. 


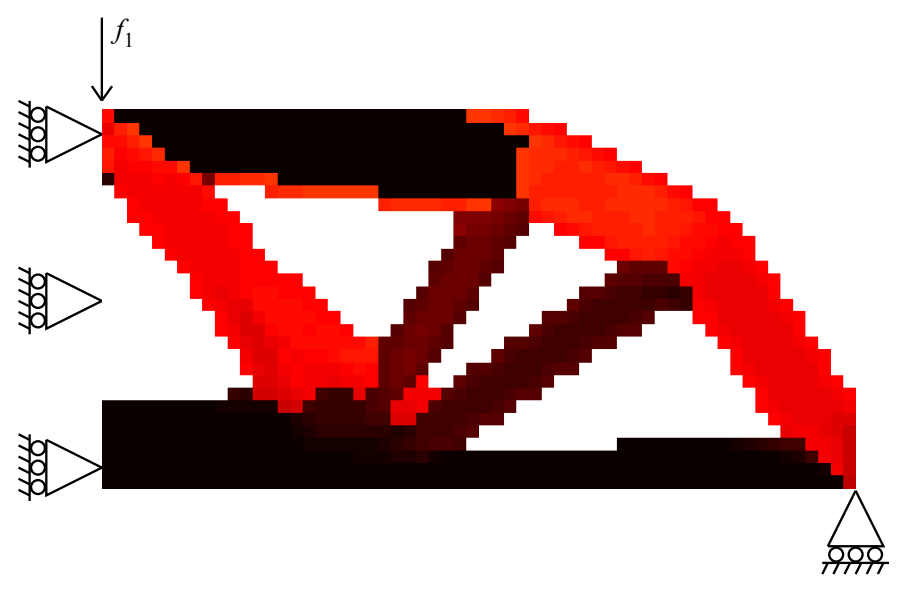

Figure 3.4: An example of using different microstructure orientations in a topology. Each unique orientation adds 1 piece of information content. In this example information content $(K)$ has been increased by 7 .

The redistribution of material in regions where local compliance is the highest is already incorporated into the SIMP method of topology optimization. However, in the code developed by Sigmund [18], there are no variables to directly control the geometric complexity of a topology. Therefore algorithms have been developed for this research that work in conjunction with Sigmund's code to add geometric complexity.

One idea of how to increase geometric complexity was to have a genetic algorithm which populated a certain number of elements in the design space with material during an iteration of the optimization [25]. This method requires the simultaneous placement of new design elements in a sequential line spanning a void for them to be of any significance in reducing compliance. The probability of such a result is extremely low. Also, genetic algorithms tend to be computationally expensive especially when coupled with topology optimization.

Yet another method tested was to periodically stop the optimization, redistribute geometry, then continue back through optimization iterations. "X-like" structures were created in the center of each void, allowing the optimizer to steer towards a more complex solution. In general this method was limited in the amount of additional complexity it added and tended to revert to the original design. 


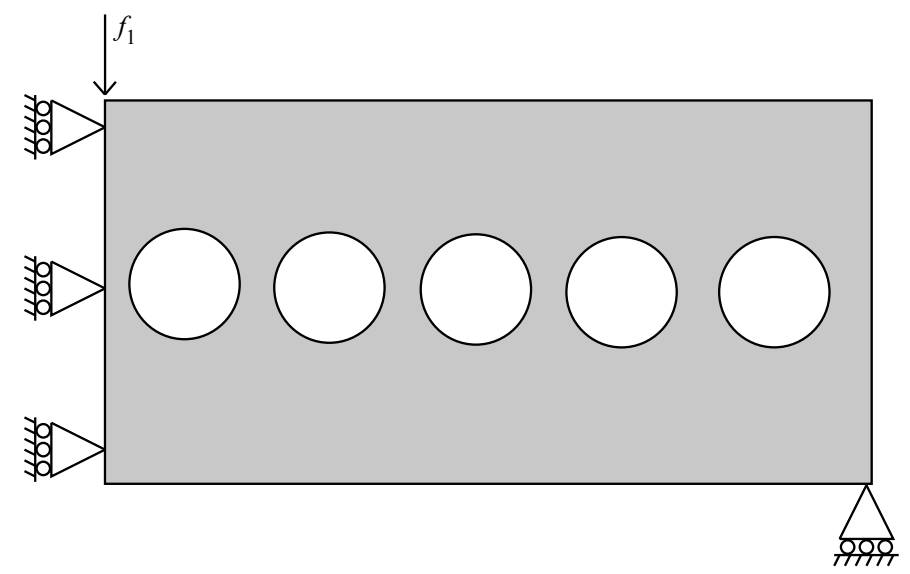

Figure 3.5: Design domain with 5 equally spaced voids in an attempt to complicate the geometry in the topology

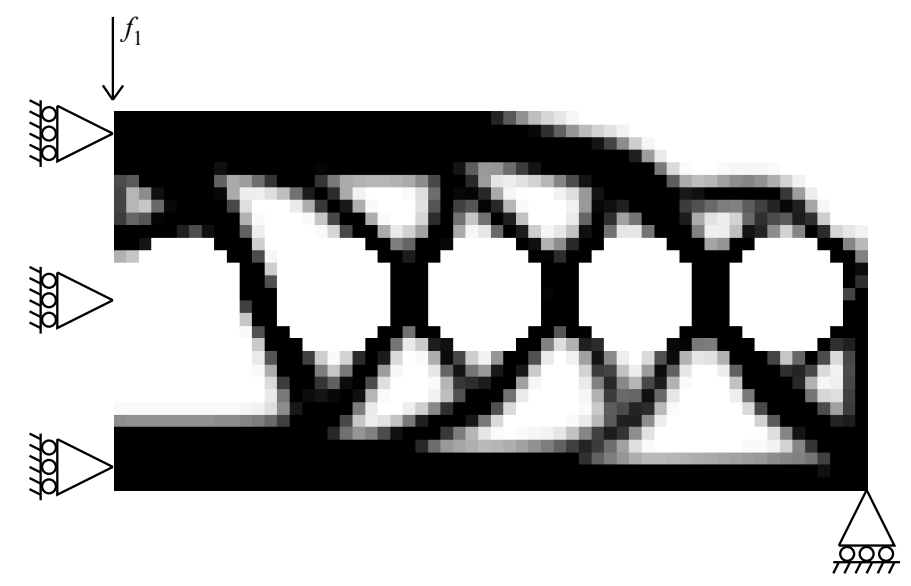

Figure 3.6: Resulting topology using the design domain in Fig. 3.5. $\bar{C}_{A}=125$ and $\bar{K}_{A}=234$. Where $\bar{C}_{A}=\left(\frac{C}{C_{A}} * 100\right)$ and $\bar{K}_{A}=\left(\frac{K}{K_{A}} * 100\right) . C$ and $K$ are evaluated from this topology, while $C_{A}$ and $K_{A}$ represent the $C$ and $K$ of the benchmark topology shown in Fig. 3.2. 
In this thesis, complicating the geometry has been accomplished by placing groups of elements in the design domain that are constrained to have zero material volume (i.e., constrained to be void regions). Creating these voids forces the topology optimization routine to navigate around them. This often results in more complex geometry. Although this approach adds complexity to the geometry inadvertently it has been the most effective and simple of the methods tested. Fig. 3.5 shows an example of voids being placed in the design domain and Fig. 3.6 shows the resulting topology after being optimized.

Using the original topology optimization result in Fig. 3.2 as a comparison, the topology in Fig. 3.6 has a $134 \%$ increase in $K$ with a $25 \%$ increase in $C$. Thus adding voids to the design domain drastically increases the information content in a resulting topology. A drawback to this method is that it decreases the size of the design domain, ensuring that the average elemental compliance $(C)$ will always be greater. This negative effect is counteracted by optimizing the microstructure orientation with the objective of minimizing $C$.

\subsubsection{Microstructure Orientation}

In this thesis, the elements within the design domain are given microstructures that have anisotropic material properties. An algorithm has been developed to find the optimal orientation of each element's microstructure to reduce the average elemental compliance $(C)$ in a topology. This simultaneously optimizes both of the optimization problem objectives. In Fig. 3.8, example results of this algorithm are shown. The $C$ in Fig. 3.8 is $22 \%$ less than that of the uniformly oriented example in Fig. 3.7 and $K$ has increased by $15 \%$.

As previously stated, using the method of adding voids to the design domain to increase $K$ will also adversely increase $C$ for isotropic materials. Thus the performance of the topology in Fig. 3.6 can easily be replicated with a different, less-complex geometry and the same volume fraction. This allows the clever engineer to make a simpler, better-performing product. Optimizing microstructure orientation resolves this issue as shown in Fig. 3.8 and Fig. 3.7. Note that even if the topology in Fig. 3.8 was created using the method of adding voids to the design domain it would still have a lower $C$ than the topology in Fig. 3.7. Thus the greatest barrier to reverse

engineering can be produced by combining both strategies: increasing geometric complexity and finding optimal microstructure orientation. 


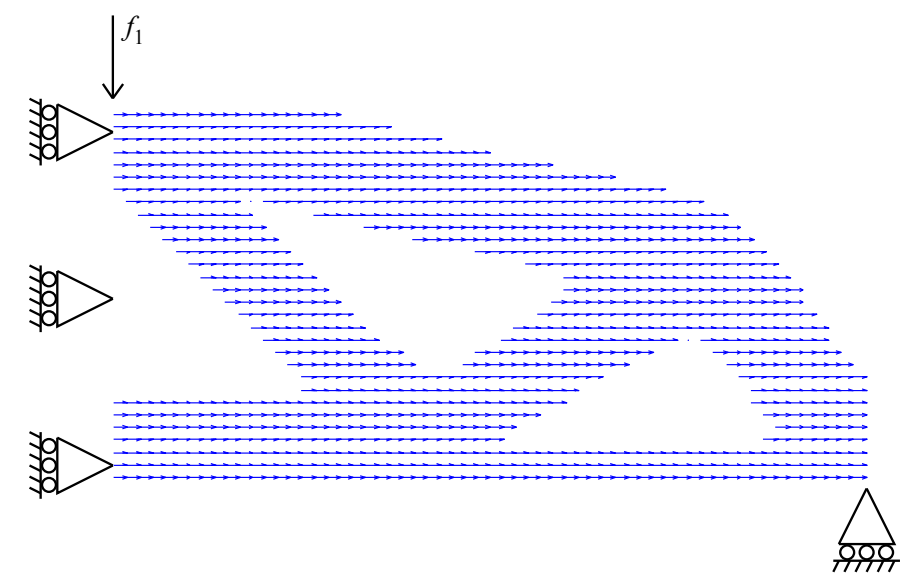

Figure 3.7: Benchmark B. A topology optimization using a uniformly oriented anisotropic material. $\bar{C}_{B}=100$ and $\bar{K}_{B}=100$. Examples using an anisotropic material will be compared to this benchmark.

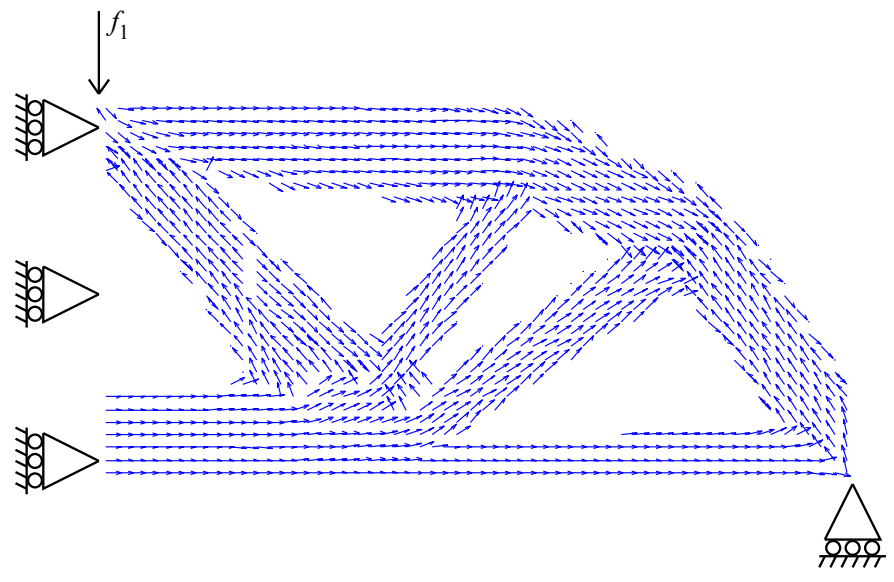

Figure 3.8: A topology optimization result using an anisotropic material that is optimally oriented at each element. $\bar{C}_{B}=78$ and $\bar{K}_{B}=115$ 


\subsection{Solving the Optimization Problem}

This section describes the algorithms that have been developed to solve the optimization problem. These algorithms (i) find the optimal size and location of a given number of voids within the design domain to maximize geometric information content, and (ii) find the optimal microstructure orientation of each element to minimize the average elemental compliance in a topology. Each is discussed in the subsections below. These algorithms work in conjunction with Sigmund's topology optimization code [18] which already incorporates the optimization problem objective of minimizing the average elemental compliance.

\subsubsection{Optimum Void Location and Size}

It has been determined that constraining groups of elements in the design domain to have no material volume (voids) often increases the geometric information content in a topology. Therefore, to maximize the information content for a prescribed number of added voids, this research has created an algorithm to find the location and size of the voids. Two approaches have been explored to find void locations that solve the optimization problem; creating void patterns and varying individual void location. The method of varying the individual void location has been chosen as the preferred method and is used in the rest of the thesis. Both methods will now be discussed.

\section{Creating Void Patterns}

Within the design domain, circles are created that are constrained to have no material volume [18]. In this approach these circles are arrayed in a pattern across the beam. The pattern is a very simple attempt to evenly space the circles through out the design space. The amount of voids in each row versus column are proportional to the size of the design space leaving the horizontal and vertical gap the same. With each addition of rows and columns the voids decrease in size. The optimizer exhaustively runs through all the different arrays of voids and produces the optimal result. In Fig. 3.9 results of this method can be seen.

Fig. 3.9 shows the optimal pattern of voids using this method. In Fig. 3.9, $C$ and $K$ of are $126 \%$ and $277 \%$ respectively of Fig. 3.2. It is also possible to weight the objectives differently so that the design with the most amount of information is selected with less regard to the minimization 


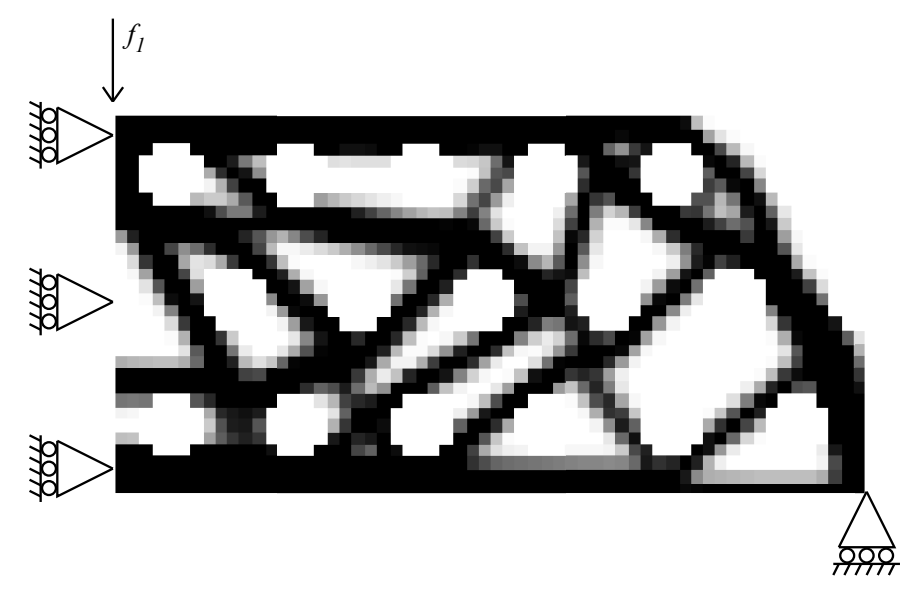

Figure 3.9: Resulting topology with optimum array of voids. $C_{a}=126$ and $K_{b}=277$

of compliance. As the preference in the optimization is weighted for decreasing compliance, the result tends to revert back to the topology without any voids. When the optimizer is weighted to increase the information content, the optimum topology becomes more like a checker board with many voids patterned across it. When this occurs it is important to recognize that patterned information is not as valuable because it is easy to reproduce.

\section{Varying Individually Void Location}

With this method a specified number of voids have freedom to change their location and radius. The optimizer takes these voids and places them to solve the optimization problem. To optimize the location of one void the Matlab function fmincon is used. The design variables are the $\mathrm{x}$ location, $\mathrm{y}$ location and radius of the void. The results of this method are shown in Fig. 3.10. The design domain was constrained as half an MBB beam with dimensions of 30 units in the $\mathrm{X}$ direction by 20 units in the $\mathrm{Y}$ direction. The $C$ and $K$ are $110 \%$ and $222 \%$ of the same size without voids.

Because of the nature of this problem finding an optimum void location for many voids simultaneously proved to be challenging. To solve this we devised an algorithm that begins by evenly spacing a designer-defined number of circular voids in the design domain. The topology optimization is then solved and the information content $(K)$ is evaluated. At this point, one void is allowed 


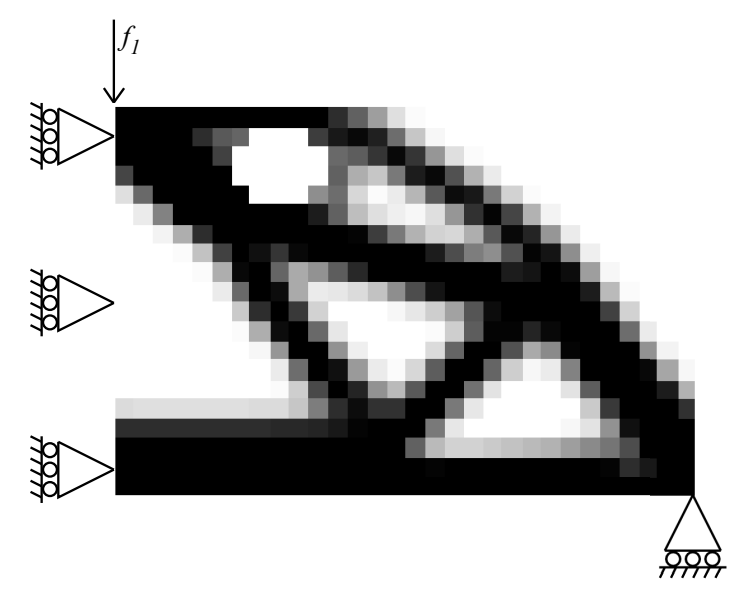

Figure 3.10: Resulting topology with optimum location of one void to maximize information content and minimize compliance

to randomly change its vertical position, horizontal position, and radius within the design domain. The random changes are determined by normally distributed random numbers, furthermore the changes are constrained to always lie within the design domain. The topology optimization is again solved and the information content for the new topology is found. This continues until (i) the information content is greater in the new topology or (ii) a specified maximum iteration is reached. The algorithm then moves to the next void and repeats the process. It continues to iterate through all the voids until it completes a cycle without finding a new topology with greater information content $(K)$, thus finding an optimum for the specified number of voids. Results of this method can be seen in Fig. 3.11. The $(K)$ in this example is $192 \%$ of that in Fig. 3.2.

\subsubsection{Optimal Microstructure Orientation}

As previously explained, each element in the design domain has a microstructure that yields anisotropic material properties. Fig. 3.12 highlights one of these elements. For the results in Chapter 3 the anisotropic material properties are such that the Young's Modulus in the 1 direction is twice that of the Young's Modulus in the 2 direction. Fig. 3.12 also illustrates how the variable $\Theta$ is able to change the orientation of the microstructure and therefore control the material properties of 


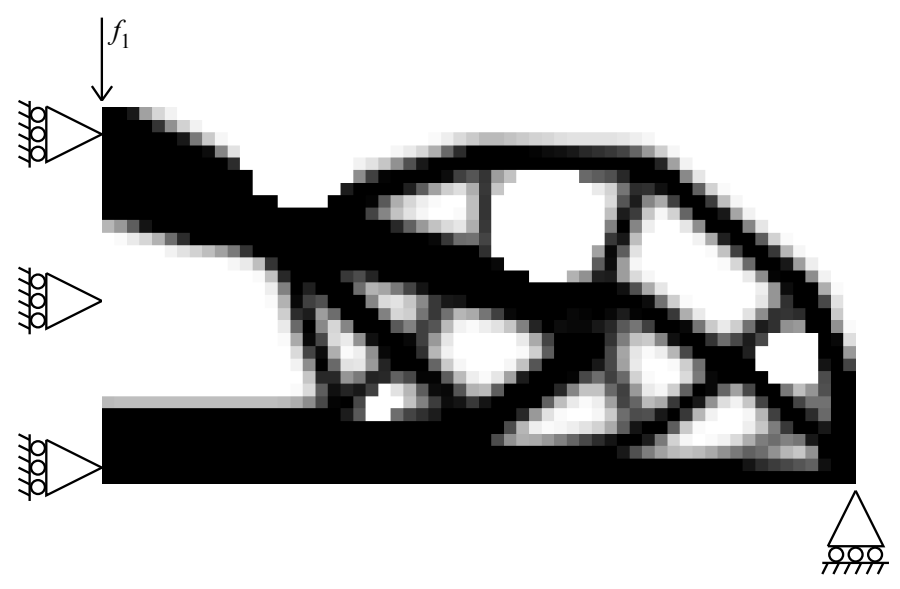

Figure 3.11: Resulting topology from using four voids and allowing them to move their location and size to find an optimum solution. $\bar{C}_{A}=134$ and $\bar{K}_{A}=192$.

each element. For $\Theta$ to accomplish this within the topology optimization it defines a transformation matrix used to transform the anisotropic constitutive matrix for each element [26].

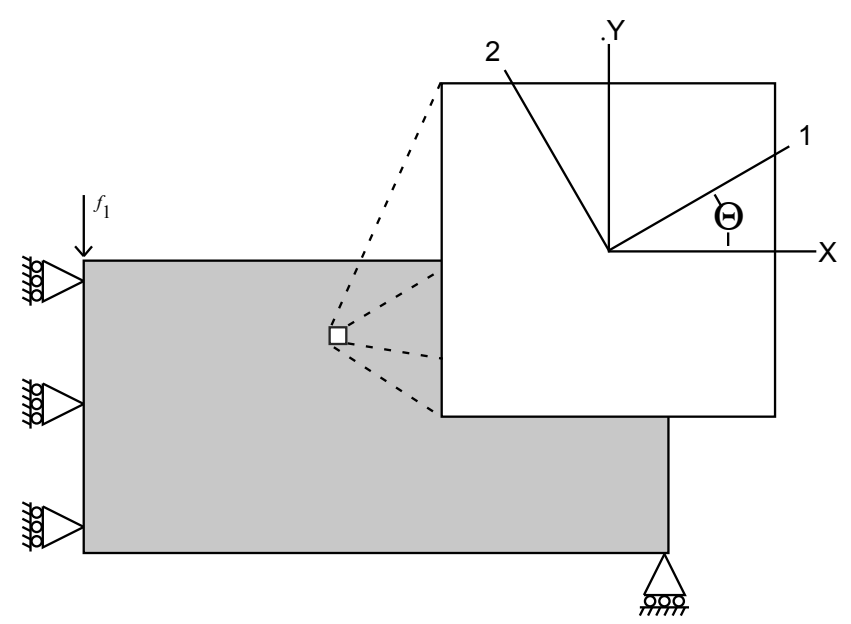

Figure 3.12: Design domain with one element highlighted. This elements microstructure orientation is determined by the value of $\Theta$.

There exists a $\Theta$ for each element that will minimize the compliance within that element. To the author's best knowledge there is not an approach in the literature for picking a $\Theta$ in each element that will minimize the element's compliance, and therefore the compliance of the whole 
topology. Therefore a method to accomplish this has been developed. Different approaches to finding this method have been tested. One idea was to use a genetic algorithm to find the location of each of the elements that would minimize the compliance [27]. This method worked, but the algorithm was extremely expensive computationally. Next, a theory was developed that the optimal value of $\Theta$ would be equal to the orientation of the highest principal stress. Since it is possible to solve for the orientation of the highest principal stress in each element, this would be very easy to combine within the topology optimization routine.

To test this hypothesis a single element was used in an exhaustive search to find the orientation of its anisotropic material properties that minimized its compliance. The test was done for 5 different loading conditions on the element; namely, pure shear, pure tension, equal and opposite loads in vertical and horizontal directions, cantilever beam with downward force on the end and a cantilever beam with a vertical and horizontal force. Fig. 3.13 shows the 5 different loading conditions on the single element and Fig. 3.14 shows the results of the test. Using FEA on the element the orientation of the principal stress was then obtained and compared to the orientation angle that was found to minimize the compliance. In all cases the stresses are equal. Therefore the hypothesis was correct and the orientation angle that minimizes the compliance is equal to the orientation angle of the highest principal stress.

Therefore to find the $\Theta$ that minimizes the average elemental compliance in the topology, $\Theta$ is set equal to the orientation angle of the maximum principal stress. The finite element routine in Sigmund's code was not sufficient to solve for the principal stresses or to develop the material properties stiffness matrix for an anisotropic material. Therefore this routine was replaced with a routine developed by Bhatti [28].

The steps to solve for $\Theta$ will now be discussed. First a two dimensional orthotropic constitutive matrix $(D)$ for the anisotropic material oriented 0 degrees off the horizontal axis is passed into the finite element routine.

$$
D=\left|\begin{array}{ccc}
D_{11} & D_{12} & 0 \\
D_{21} & D_{22} & 0 \\
0 & 0 & D_{33}
\end{array}\right|
$$



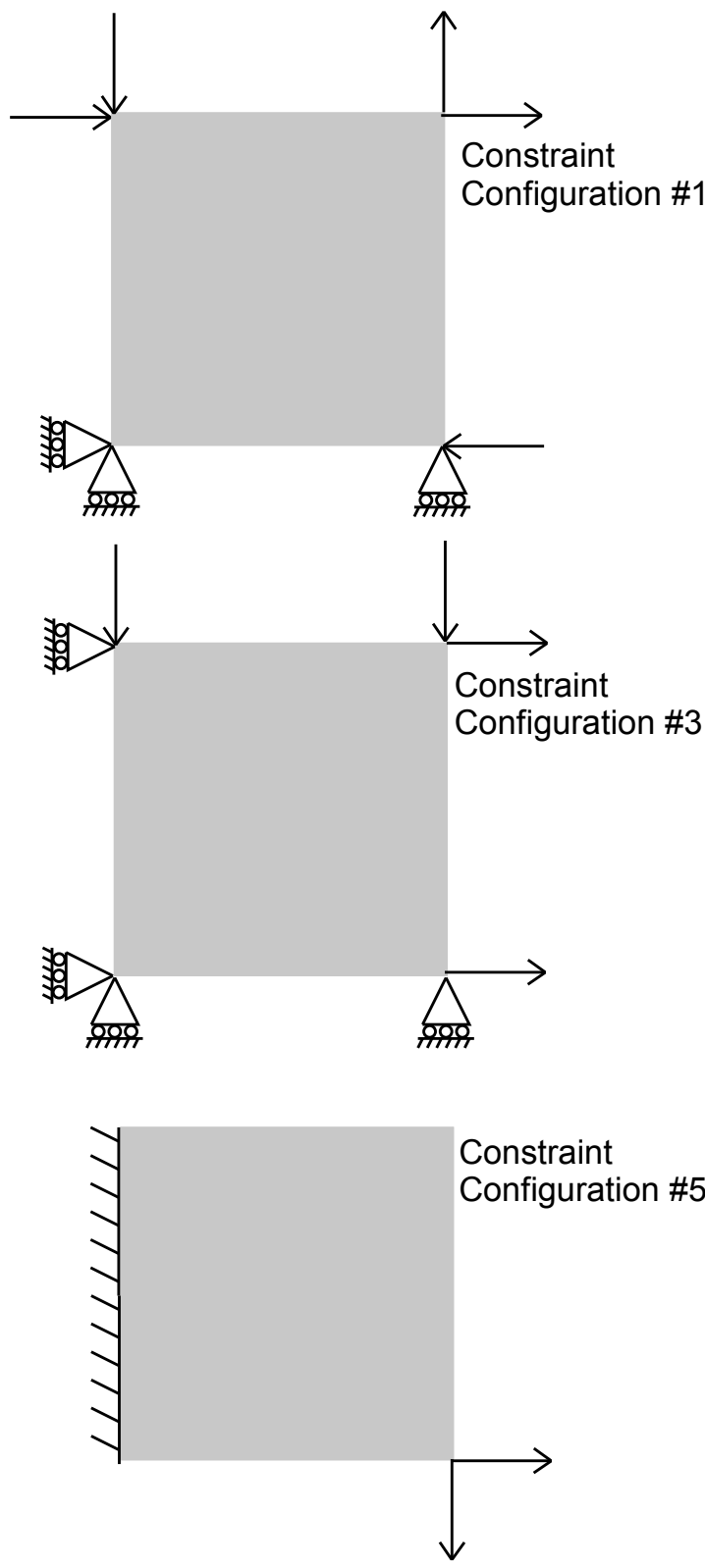
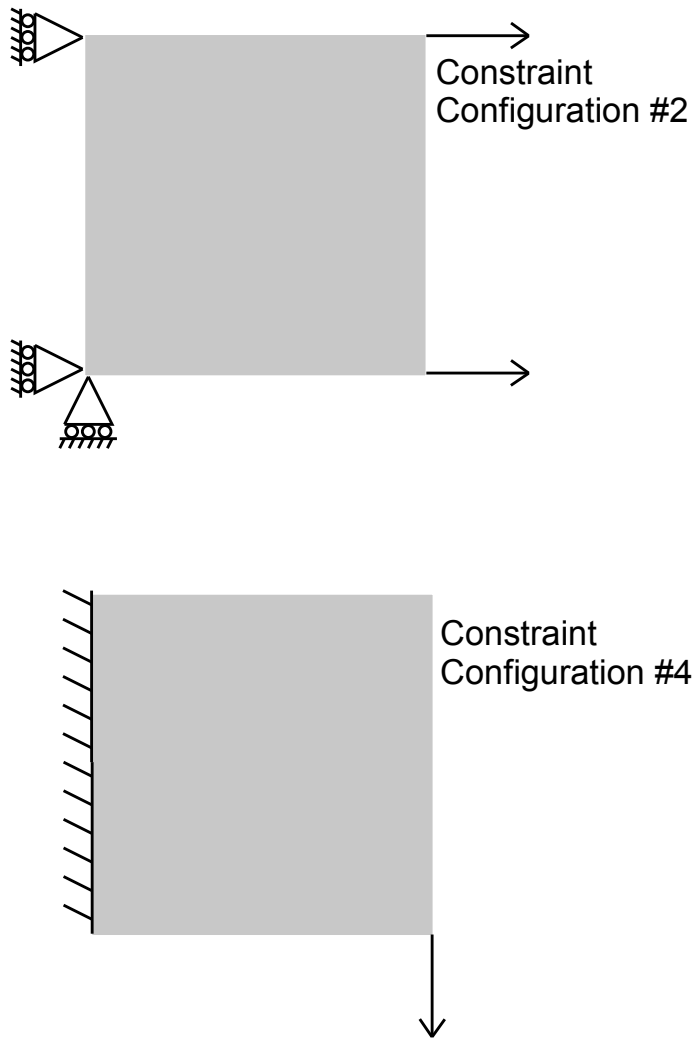

Figure 3.13: 5 elements with loads and boundary conditions used for testing the compliance at different microstructure orientations

$$
\begin{gathered}
D_{11}=E_{1} /\left(1-v_{12} v_{21}\right) \\
D_{12}=D_{11} v_{12}
\end{gathered}
$$



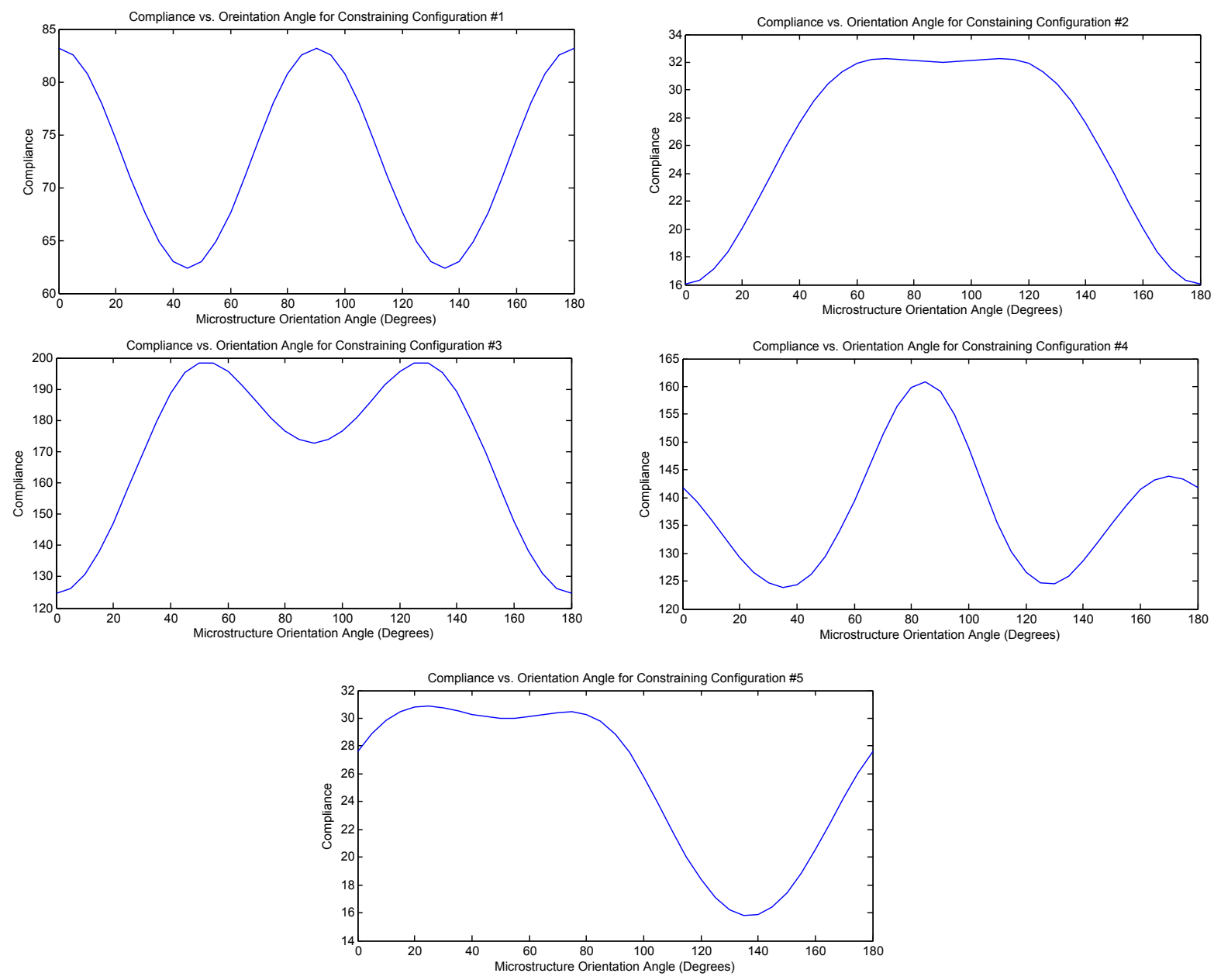

Figure 3.14: These graphs represent the compliance vs. orientation for the elements shown in Fig. 3.13

$$
\begin{gathered}
D_{21}=D_{22} v_{21} \\
D_{22}=E_{2} /\left(1-v_{12} v_{21}\right) \\
D_{33}=G_{12}
\end{gathered}
$$

Using these material properties a iteration of the topology optimization routine is then completed. Within this routine the displacement vector for each element $(U)$ is calculated. The 
stress vector $(S)$ is then solved for each element using the equation

$$
S=D B U
$$

where $B$ is the element shape functions matrix and $U$ is the displacement vector for each element. The stress vector $(S)$ is then used to find the principal stresses and their orientations. The maximum principal stress's orientation for each element is selected and replaces the value of $\Theta$ in each element. The $\Theta$ is then used within the transformation matrix $T$ to solve for the new constitutive matrix $\left(D_{\text {new }}\right)$.

$$
T=\left|\begin{array}{ccc}
\cos ^{2}(\Theta) & \sin ^{2}(\Theta) & 2 \cos (\Theta) \sin (\Theta) \\
\sin ^{2}(\Theta) & \cos ^{2}(\Theta) & -2 \cos (\Theta) \sin (\Theta) \\
-\sin (\Theta) \cos (\Theta) & \cos (\Theta) \sin (\Theta) & \left(\cos ^{2}(\Theta)-\sin ^{2}(\Theta)\right)
\end{array}\right|
$$

$D_{\text {new }}$ replaces $D$ and Topology optimization performs another iteration. Therefore the optimization of the microstructure orientation has been fully integrated into the topology optimization routine. 


\section{CHAPTER 4. EXAMPLES}

This Chapter demonstrates the method described in Chapter 3 being used the design of a novel electrical contact for a consumer electronics product. This example shows that the methods developed in this thesis can increase the time to reverse engineer this product.

\subsection{Case Study: Electrical Contact for a Consumer Electronics Product}

This section demonstrates how the methods introduced in this thesis can be used for the design of a novel electrical contact for a consumer electronics product. Many electrical contacts are manufactured through progressive die forming. As such, many contacts have large width to height ratios. Given the market demand for miniaturization of electronics, some electrical contact manufactures are beginning to explore the benefits of contacts with very low width to height ratios. This section describes such a contact, and shows that topology optimization can be used to identify a contact topology that could be fabricated from a planar sheet of copper and require no die forming, thus simplifying the manufacturing to a blanking process. Validating the financial benefit of this simplification is not the focus of this thesis.

To function properly from an electrical point of view, electrical contacts require a certain contact normal force for a given deflection. The design requirements for this example are that the contact must have a deflection between $0.35 \mathrm{~mm}$ and $1 \mathrm{~mm}$ for a contact normal force of $1 \mathrm{~N}$. Also for this example, designs with a deflection closer to $0.35 \mathrm{~mm}$ are preferred. In this design, a cube textured copper material is used. This material has been chosen because of the large variation of its material properties associated with its different microstructure orientations [29].

The design domain with the boundary conditions and loads are defined as shown in Fig. 4.1. This design domain has a horizontal dimension of $25 \mathrm{~mm}$ and a vertical dimension of $10 \mathrm{~mm}$. The downward force is $1 \mathrm{~N}$, and the horizontal force is $0.5 \mathrm{~N}$ (simulating the maximum horizontal force seen by the electrical contact during connection). The electrical contact is fixed on the lower half 
of its left side in all degrees of freedom. Executing the topology optimization routine based on this design domain results in the topology shown in Fig. 4.2. Note that for this case, the number of unique microstructures is constrained to be 1. This first design (Case 1) of the electrical contact will be used as a benchmark to the remaining examples.

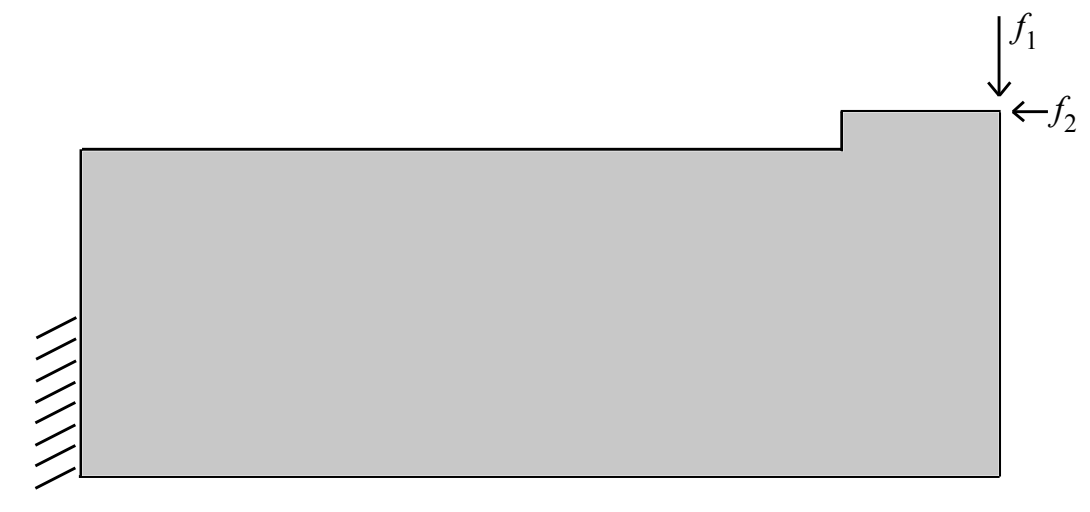

Figure 4.1: Design domain for the novel electrical contact

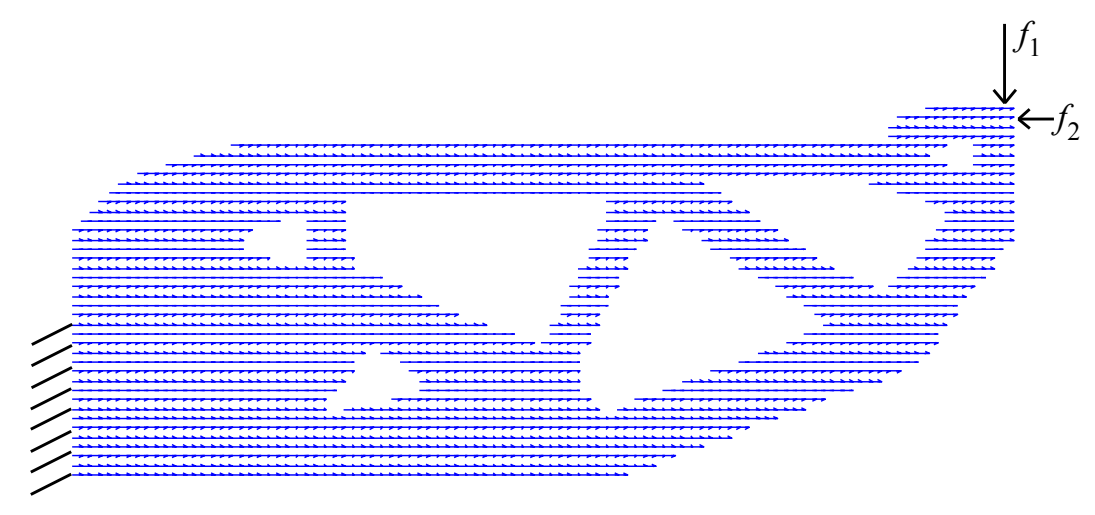

Figure 4.2: Case 1:(Benchmark C) Uniformly oriented Topology with no forced voids. $\bar{C}_{C}=100$ and $\bar{K}_{C}=100$.

The next three designs show the results of the algorithms developed in this thesis working in conjunction with the topology optimization routine to maximize $K$ and minimize $C$. Case 2 (shown 
in Fig. 4.3) is a design of the electrical contact after 4 voids have been optimally located and sized within the design domain using the method described in section 3.4.1. Within the algorithm, the voids random changes in location and size are determined from a normal distribution with a mean of 0 and a standard deviation of $5 \%$ of the design domain. For Case 2, one unique microstructure is allowed. The voids in Case 4 are created the same way. Case 3 (shown in Fig. 4.4) is a design of the electrical contact where the microstructure of each element in the design domain has been optimally oriented. Case 4 (shown in Fig. 4.5) is a design where 4 voids have been optimally located and sized within the design domain (as described in Case 2), and the microstructure of each element in the design domain has been optimally oriented.

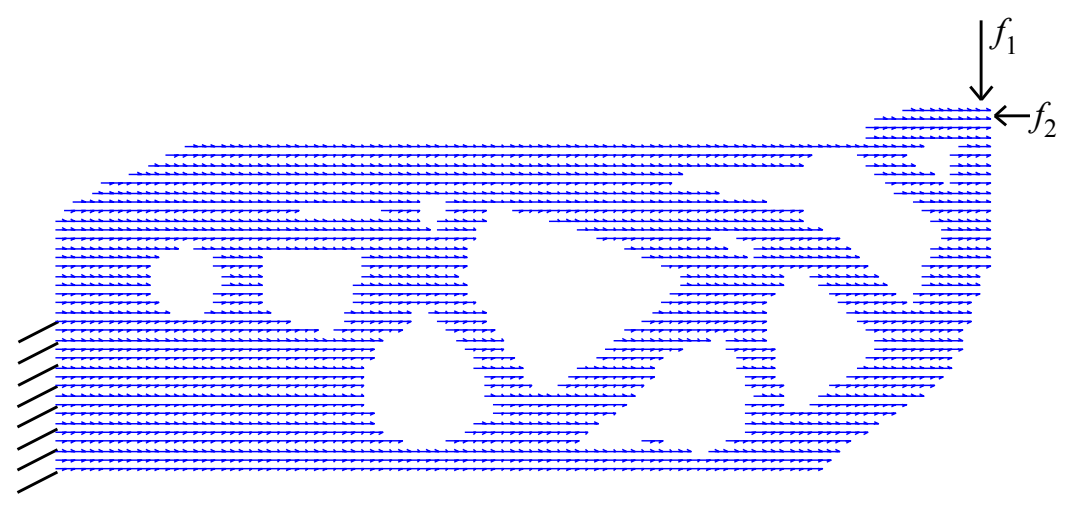

Figure 4.3: Case 2: Uniformly oriented topology with 4 forced voids that have been optimally located and sized. $\bar{C}_{C}=109.5$ and $\bar{K}_{C}=192$.

In Table 4.1 a comparison of information content and the deflection of the electrical contact can be seen for each case. Case 2 (compared to the benchmark design) has an increased information

Table 4.1: Comparison of information content $(K)$ and deflection in the different cases

\begin{tabular}{|c|c|c|}
\hline \hline Example & Info. Content & Deflection $(\mathrm{mm})$ \\
\hline Case 1 (benchmark) & 44 & 0.53 \\
\hline \hline Case 2 & 77 & 0.58 \\
\hline Case 3 & 58 & 0.375 \\
\hline Case 4 & 98 & 0.45 \\
\hline \hline
\end{tabular}




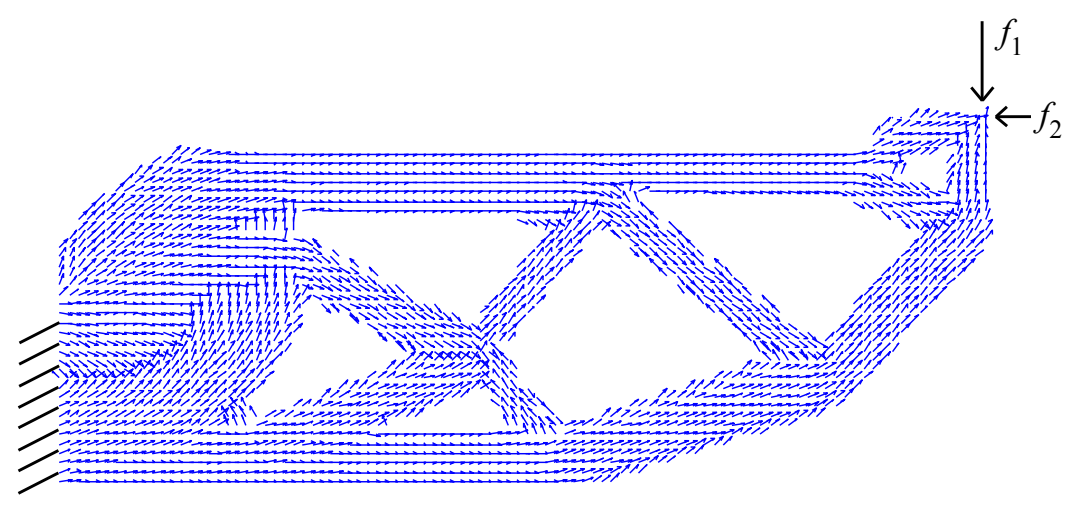

Figure 4.4: Case 3: Optimally oriented topology with no forced voids initially in the design space. $\bar{C}_{C}=71$ and $\bar{K}_{C}=132$.

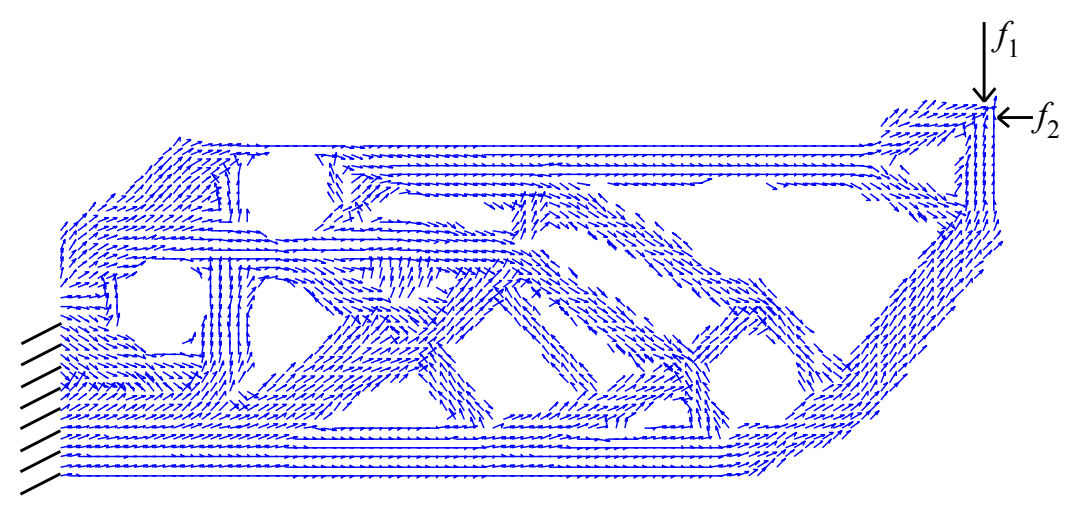

Figure 4.5: Case 4: Optimally oriented topology with 4 forced voids that have been optimally located and sized. $\bar{C}_{C}=85$ and $\bar{K}_{C}=223$.

content $(K)$, but the deflection has undesirably increased. Although this design would be more difficult to reverse engineer, a clever engineer would be able to create a simpler beam using less material that had the same deflection; resulting in a better, less-expensive product. Although this risk is present when using the design in Case 2, the time to reverse engineer the product has been increased.

Case 3 has the lowest deflection (which is desirable) out of the 4 designs. Also, compared to the benchmark the $K$ has increased. This shows that by manipulating the microstructure, a designer 
is able to increase the time to reverse engineering a product while simultaneously improving the product's performance. This design has the absolute lowest possible defection for the given design domain. However, the information content, $K$, can still be drastically increased.

Case 4 has the greatest amount of $K$ and a desirably low deflection. Even though its deflection is not as low as the deflection in Case 3, it is lower then the deflection found in the benchmark design. Thus, it has a better performance than the benchmark design and a much higher information content. Therefore Case 3 and Case 4 are able to increase the time to reverse engineering a product without giving up the desirable structural characteristic of compliance. Although Case 4 has a slight increase in deflection over Case 3, it has a drastic increase in $K$, potentially making it the superior design. A CAD model of this design can be seen in Fig. 4.6 and Fig. 4.7.

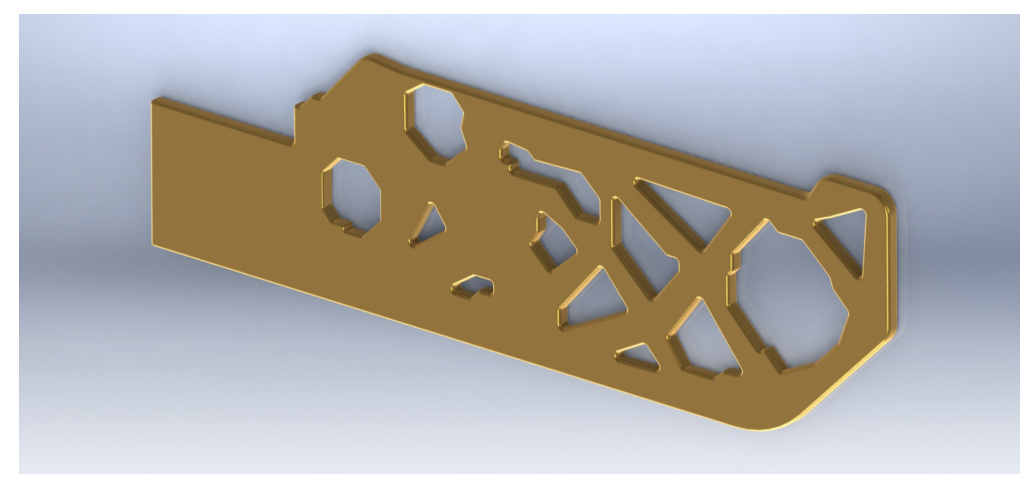

Figure 4.6: 3D Model of the contact design in Case 4

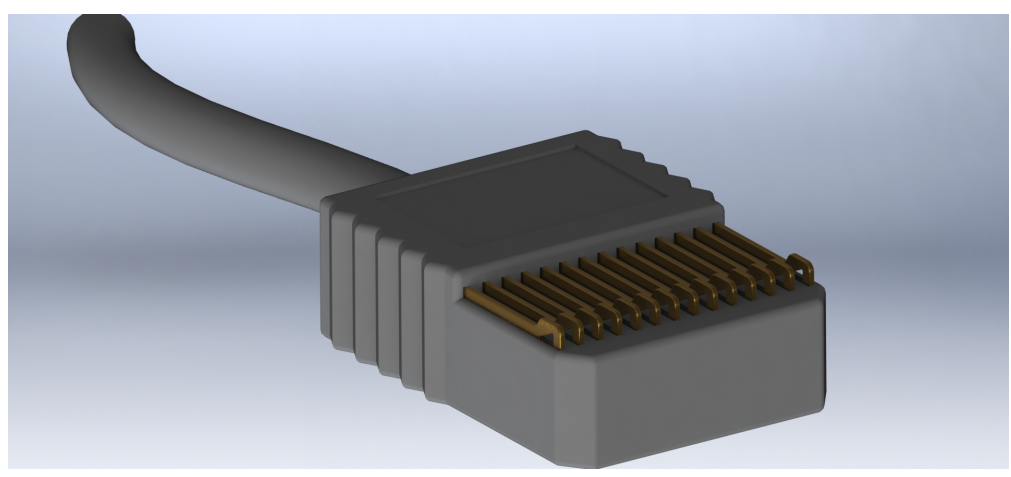

Figure 4.7: 3D Model of a connector using the contact design in Case 4 


\section{CHAPTER 5. CONCLUSIONS AND FUTURE WORK}

\subsection{Conclusion}

This thesis has shown that the tool of topology optimization can be used to identify topologies that are both time-consuming to reverse engineer and structurally desirable. The algorithms introduced in this thesis enable topology optimization to be used in this way. Specifically, an algorithm is presented that automatically evaluates the information content of any two-dimensional topology. This evaluation allows an additional objective, the maximization of information content, to be added to traditional topology optimization objectives. To make the inclusion of this objective more meaningful, microstructure sensitive design is also folded into the process by allowing individual topology elements to be optimally oriented. Various test cases are performed in this thesis. Using isotropic materials and maximizing information content results in an undesirable increase in structural compliance. When using anisotropic materials, minimizing structural compliance and maximizing information content can occur desirably without significant compromise. This comes from increasing the information content held in both the geometry and microstructure of a topology. An electrical contact for a consumer product is also examined. Because the topology optimization is able to examine numerous complicated contact designs in search of one that would out perform traditional isotropic progressive-die formed contacts, a contact is found that competitors would want to reverse engineer, yet have significant difficulty doing so.

\subsection{Future Work}

While fulfilling the objectives of this research, new areas of research have been suggested. Some of these are discussed below.

In calculating the information content contained in a product, it became difficult to compare the information content in the geometry and the information content in the microstructure. 
These two categories of information are noticeably different and the concept of weighing the two information types relative to each other could be explored.

Optimizing the microstructure in every element has been a very effective way to minimize the compliance in our examples. The problem is that there is no common manufacturing techniques to create such structures. More research to create such manufacturing processes would be an incredible advance for structural applications.

This thesis has shown that the time to reverse engineer a product can be increased using the tool of topology optimization without giving up the desirable structural characteristic of compliance. Other ways to automatically improve barriers to reverse engineer could be explored. 


\section{REFERENCES}

[1] Samuelson, P.;Scotchmer, S., 2001. "Law and economics of reverse engineering, the." Yale $L J, 111$, p. 1575.1

[2] McLoughlin, I., 2008. "Secure embeddeed systems: the threat of reverse engineering." Parallel and Distributed Systems, 2008. ICPADS'08. 14th IEEE International Conference on, pp. 729-736. 1

[3] Grand, J., 2004. "Practical secure hardware design for embedded systems." Proceedings of the 2004 Embedded Systems Conference. 1

[4] Curtis, S., Harston, S., and Mattson, C., 2011. "The fundamentals of barriers to reverse engineering and their implementation into mechanical components." Research in Engineering Design, pp. 1-17. 1

[5] Harston, S., Mattson, C., and Adams, B., 2010. "Metrics for evaluating the barrier and time to reverse engineer a product." Journal of Mechanical Design, 132, p. 9. 1, 2, 10

[6] Reed, Richard;Defillippi, R. J., 1990. "Causal ambiguity, barriers to imitation, and sustainable competitive advantage." The Academy of Management Review, 15, pp. 88-102. 2

[7] Bendsoe, M. P., and Sigmund, O., 2004. Topology optimization: theory, methods and applications. Springer. 5

[8] Sigmund, O.; Torquato, S., 1997. "Design of materials with extreme thermal expansion using a three-phase topology optimization method." Journal of the Mechanics and Physics of Solids, 45, pp. 1037-1067. 5

[9] Zhou, H., and Killekar, P. P., 2011. "The modified quadrilateral discretization model for the topology optimization of compliant mechanisms." Journal of Mechanical Design, 133(11), p. 111007.5

[10] Kota, S., Joo, J., Li, Z., Rodgers, S., and Sniegowski, J., 2001. "Design of compliant mechanisms: Applications to mems." Analog Integrated Circuits and Signal Processing, 29, pp. 715. 5

[11] Lee, S., and Tovar, A., 2013. "Topology optimization of piezoelectric energy harvesting skin using hybrid cellular automata." Journal of Mechanical Design, 135(3), p. 031001. 5

[12] Nomura, T., Nishiwaki, T., Nishiwaki, S., Sato, K., and Hirayama, K., 2009. "Topology optimization for the design of periodic microstructures composed of electromagnetic materials." Finite Elements in Analysis and Design, 45, pp. 210-226. 5 
[13] Rakshit, S., and Ananthasuresh, G., 2008. "Simultaneous material selection and geometry design of statically determinate trusses using continuous optimization." Structural and Multidisciplinary Optimization, 35, pp. 55-68. 5

[14] Li, Q., Steven, G. P., Querin, O. M., and Xie, Y., 1999. "Shape and topology design for heat conduction by evolutionary structural optimization." International Journal of Heat and Mass Transfer, 42(17), pp. 3361 - 3371. 5

[15] Torquato, S., Hyun, S., and Donev, A., 2002. "Multifunctional composites: Optimizing microstructures for simultaneous transport of heat and electricity." Phys. Rev. Lett., 89, Dec, p. 266601.5

[16] Bendsoe, M., 1989. “Optimal shape design as a material distribution problem." Structural optimization, 1, pp. 193-202. 5

[17] Rozvany, G., 2009. "A critical review of established methods of structural topology optimization." Structural and Multidisciplinary Optimization, 37, pp. 217-237 10.1007/s00158-0070217-0. 5

[18] Sigmund, O., 2001. "A 99 line topology optimization code written in matlab." Structural and Multidisciplinary Optimization, 21, pp. 120-127. 6, 9, 10, 14, 18

[19] Challis, V., 2010. “A discrete level-set topology optimization code written in matlab.” Structural and Multidisciplinary Optimization, 41, pp. 453-464 10.1007/s00158-009-0430-0. 6

[20] Yamada, T., Izui, K., and Nishiwaki, S., 2011. "A level set-based topology optimization method for maximizing thermal diffusivity in problems including design-dependent effects." Journal of Mechanical Design, 133(3), p. 031011. 6

[21] Adams, B. L., Kalidindi, S. R., Fullwood, D. T., and Niezgoda, S. R., 2010. "Microstructure sensitive design for performance optimization.” Progress in Materials Science, 55(6), pp. 477 $-562.6,7$

[22] Harston, S., Mattson, C., and Adams, B., 2010. "Capitalizing on heterogeneity and anisotropy to design desirable hardware that is difficult to reverse engineer." Journal of Mechanical Design, 132, p. 11.6

[23] Messac, A.;Mattson, C., 2002. "Generating well-distributed sets of pareto points for engineering design using physical programming." Optimization and Engineering, 3, pp. 431-450. 10

[24] Balling, R., 1999. "Design by shopping: A new paradigm?.” Proceedings of the Third World Congress of Structural and Multidisciplinary Optimization (WCSMO-3), 1, pp. 295-297. 10

[25] Saitou, K.;Jakiela, M., 1994. "Genetic algorithms as an approach to configuration and topology design.” Journal of Mechanical Design, 116, p. 1005. 14

[26] Li, S., and Atluri, S., 2008. "The mlpg mixed collocation method for material orientation and topology optimization of anisotropic solids and structures." CMES: Computer Modeling in Engineering \& Sciences, 30(1), pp. 37-56. 21 
[27] Harston, S., Mattson, C., and Koecher, M., 2010. "A topology optimization method with anisotropic materials-13th aiaa/issmo multidisciplinary analysis optimization conference multidisciplinary analysis optimization conferences." In American Institute of Aeronautics and Astronautics. 22

[28] Bhatti, M. A., 2005. Fundamental finite element analysis and applications. John Wiley \& Sons. 22

[29] Takahashi, R., Prasai, D., Adams, B. L., and Mattson, C. A., 2012. "Hybrid bishop-hill model for elastic-yield limited design with non-orthorhombic polycrystalline metals." Journal of Engineering Materials and Technology, 134(1), p. 011003. 27 